\title{
Endocarditis with aneurysm involving an aortic homograft used to correct a truncus arteriosus: medical-surgical salvage
}

\author{
Mary Allen Engle, Samuel J Lang, Paul Edelson
}

\begin{abstract}
An aneurysm of an aortic homograft conduit, used to correct a type I truncus arteriosus anomaly in a four month old infant, developed when the patient was 15. Blood cultures grew Staphylocuccus aureus. The aneurysm was detected by magnetic resonance imaging and digital subtraction angiocardiography. An emergency open heart operation, guided by these investigations, was performed to remove the original homograft and replace it with another valved aortic homograft. Postoperative antibiotic treatment had to be stopped when profound neutropenia developed. This responded to treatment with recombinant human granulocyte colony stimulating factor.

Three years later she was symptom free and did not require medication. Chest $x$ rays and echocardiograms showed a normally functioning heart and conduit valve.
\end{abstract}

We report the successful outcome of two lifethreatening events 15 years after placement of an aortic homograft conduit to repair a truncus arteriosus.

\footnotetext{
Division of Pediatric Cardiology, The New York HospitalCornell University Medical Center, New York, USA M A Engle

Division of Cardiothoracic Surgery, The New York HospitalCornell University Medical Center, New York, USA

S J Lang

Division of Infectious Disease, The New York Hospital-Cornell University Medical Center, New York, USA

P Edelson

Correspondence to Professor Mary Allen Engle, Division of Pediatric Cardiology, The New York Hospital, 525 E 68th Street New York, NY 10021, USA.
}

\section{Case report}

HISTORY

An aortic homograft and closure of the ventricular septal defect were used to repair a type I truncus arteriosus in a four month old baby who was born prematurely and weighed $1390 \mathrm{~g}$ at birth and $3 \mathrm{~kg}$ at surgery. ${ }^{1}$ She did well under regular cardiological follow up, though the conduit became calcified and systolic and diastolic murmurs of conduit valve stenosis and regurgitation developed. Cardiac catheterisation when she was three years old and seven years old showed gradients across the homograft of 28 and $25 \mathrm{~mm} \mathrm{Hg}$ respectively between the right ventricle and pulmonary artery. Doppler echocardiography at 10 and 13 years showed a systolic pressure drop of $44 \mathrm{~mm} \mathrm{Hg}$ along the conduit. Magnetic resonance imaging at 12 years displayed flow through the conduit.

PRESENT ILLNESS

At the age of 15 she had fever for one week, which did not respond to oral erythromycin. Examination showed splenomegaly and the systolic and early diastolic murmurs noted earlier. The cardiac contour and size as well as pulmonary vascularity and parenchyma were normal on the radiograph. Continuous wave Doppler echocardiography showed a pressure drop of $100 \mathrm{~mm} \mathrm{Hg}$ at the proximal end of the conduit. Blood cultures grew Staphylococcus aureus that was resistant to erythromycin but sensitive to nafcillin, gentamicin, and vancomycin.

\section{COURSE}

Despite treatment with intravenous nafcillin and gentamicin, temperature spikes persisted each afternoon. Over the next four days she developed bilateral pleural effusions, pericardial effusion, and signs of right-sided cardiac failure. Digoxin and diuretics were added to the regimen. Three days later a chest $x$ ray showed nodular densities in both lung fields and a rounded prominence in the region of the outflow tract of the right ventricle that rapidly increased in size. A computed tomographic scan of the chest, with contrast enhancement, identified pleural and pericardial effusions as well as infiltrates in the lower lobes. Digital subtraction angiocardiography (DSA) (fig 1) and magnetic resonance imaging (MRI) (fig 2)

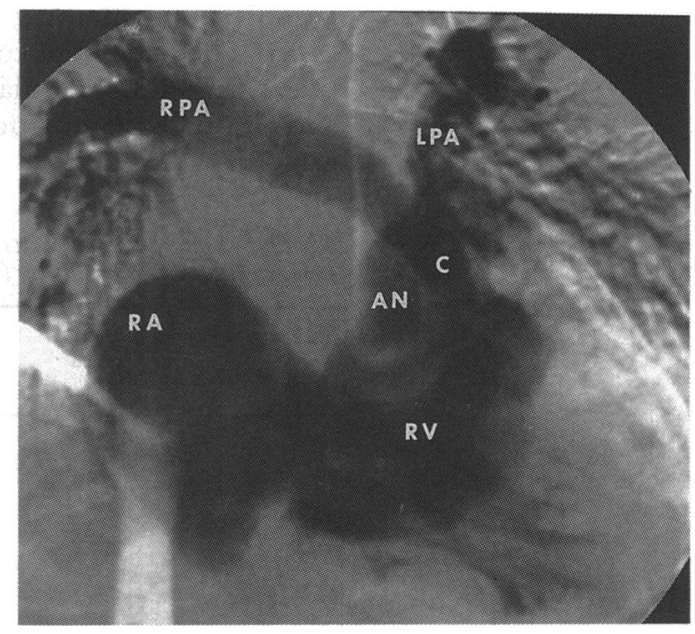

Figure 1 Digital subtraction angiocardiogram showing the aneurysm $(A N)$ superimposed on the conduit $(C)$. The aneurysm continued to the left and right pulmonary arteries ( $L P A, R P A)$ and the right atrium $(R A)$ and ventricle $(R V)$ were dilated. 
Figure 2 Magnetic resonance imaging (axial view) showing maximal dilatation of the aneurysm $(A N)$ and its relation to the overlying sternum and to the aortic root $(A o)$ and the right and left atria ( $R A$ and $L A$ ). An arrow points to the infarct. Pleural effusions were present.

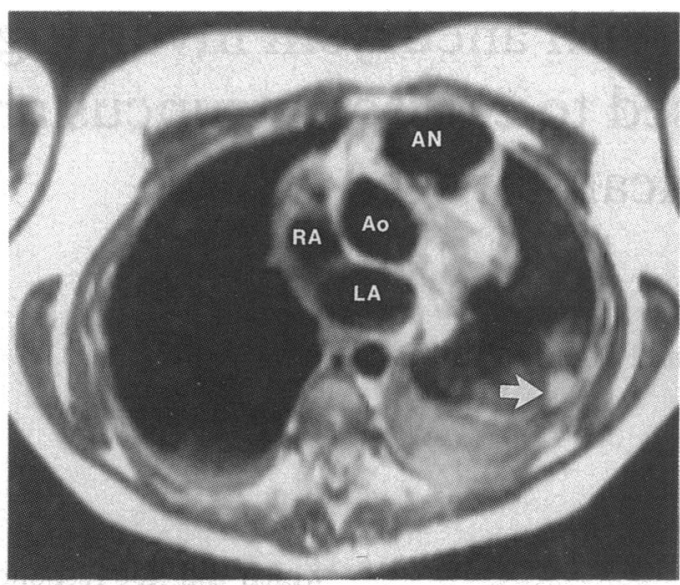

confirmed the suspicion of aneurysm where the conduit connected with the right ventricle and of haemorrhagic infarcts in both lung fields with bilateral pleural effusions.

SURGERY

Open heart surgery was undertaken as an emergency to remove the aneurysm and the original homograft and replace it with another valved aortic homograft. Because MRI and DSA had shown that the aneurysm was contiguous anteriorly with the sternum (fig 2) a right thoracotomy was used for cannulation of the venae cavae and aorta. When the median sternotomy incision was reopened and the sternum divided, the aneurysm was indeed entered, but cardiopulmonary bypass was immediately started and bleeding controlled. The thin walled aneurysm originated from the right ventricle proximal to the graft, which was obstructed by granulation tissue proximally but was patent and densely calcified. The conduit was removed and the pulmonary artery branches were enlarged to accommodate the new, size 21 cryopreserved aortic homograft. Systolic pressure measured in the right ventricle when the patient's condition was stable off bypass was less than half systemic pressure. Microscopy of the removed graft showed calcified, focally necrotic connective tissue containing many Gram positive cocci. The aneurysm contained dense connective tissue and granulation tissue with acute and chronic inflammation. Cultures yielded no growth.

Relation of white blood counts to operation and treatment with granulocyte stimulating factor (GSF)

\begin{tabular}{|c|c|c|c|}
\hline $\begin{array}{l}\text { Day } \\
\text { postop }\end{array}$ & 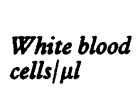 & 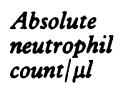 & $\begin{array}{l}\text { Day of } \\
\text { GSF }\end{array}$ \\
\hline $\begin{array}{r}0 \\
3 \\
6 \\
7 \\
8 \\
9 \\
10 \\
12 \\
13 \\
14 \\
15 \\
16 \\
17 \\
18 \\
19\end{array}$ & $\begin{array}{c}14600 \\
11100 \\
4500 \\
3100 \\
1200 \\
1000 \\
600 \\
400 \\
600 \\
1000 \\
2600 \\
4000 \\
4700 \\
10900 \\
7300\end{array}$ & $\begin{array}{r}12100 \\
10700 \\
4100 \\
2480 \\
620 \\
160 \\
0 \\
0 \\
0 \\
0 \\
30 \\
1200 \\
3290 \\
8070 \\
5620\end{array}$ & $\begin{array}{l}0 \\
1 \\
2 \\
3 \\
4 \\
5 \\
6 \\
7 \\
8\end{array}$ \\
\hline
\end{tabular}

POSTOPERATIVE COURSE

Treatment with nafcillin and gentamicin was continued. On the third postoperative day when a rash appeared, intravenous amikacin replaced gentamicin. Neutropenia began to develop on the sixth day (table); so nafcillin was stopped and vancomycin started. Three days later, oral ciprofloxacin was added. Her temperature rose intermittently to $39.6^{\circ} \mathrm{C}$. Neutropenia became profound: total white blood cells $400-600 / \mu 1$ with absolute neutrophil counts of 0 (table). A bone marrow biopsy specimen was markedly hypocellular, with fully maturing erythroid elements and megakaryocytes but virtual absence of myeloid elements. The peripheral blood did not contain neutrophil antibodies.

We obtained permission from the Food and Drug Administration, from the manufacturers (AMGEN), and from the Institutional Committee on Human Rights in Research for the compassionate use of recombinant human granulocyte colony stimulating factor (rhGCFS). ${ }^{2}$ Recombinant rhG-GSF in a daily dose of $3 \mu \mathrm{g} / \mathrm{kg}$ per day was chosen because of previous experience in children with congenital or chemotherapy-induced neutropenic disorders. ${ }^{23}$ This agent was administered subcutaneously for 14 successive days. The white blood cell count began to increase (table) to a normal number of white blood cells and neutrophils by the seventh day of treatment with rhG-CSF. After two weeks the count reached $15800 / \mu \mathrm{l}$. As the count rose she became afebrile and continued so. After the fifth day without fever she was discharged; she was treated oral ciprofloxacin for two more weeks.

In the next three years she remained symptom free and did not require medication. Chest $x$ ray showed that the size and contour of the heart were normal, and echocardiography showed normal function of the myocardium and conduit valve.

\section{Discussion}

This patient had endocarditis with a rapidly expanding aneurysm, considerable obstruction to right ventricular outflow, and emergency cardiac surgery. She also had another life-threatening complication-a severe reaction to one or more of the intravenous antibiotics needed to control the infection.

When the aneurysm appeared and expanded during the course of antibiotic treatment, two non-invasive diagnostic tests (DSA and MRI) showed the proximity of the aneurysm to the overlying sternum and this information helped us to plan the life-saving operation. A review of 143 episodes of endocarditis in children between 1950 and 1988 noted that 23 patients needed cardiac surgery, seven of these for resections of infected areas, chiefly related to the ductus or coarctation of the aorta. ${ }^{4}$

When profound neutropenia appeared we sought permission to use a regulatory glycoprotein that stimulates neutrophil granulocyte production from committed 
haematopoietic progenitor cells both in vitro and in vivo. In addition it modulates multiple differential functions of mature neutrophils. Thus this agent enabled her neutrophils to recover and to function.

Medical and surgical management enabled a patient as an infant to survive not only considerable prematurity but also open heart surgery by a new procedure (to construct a pulmonary artery conduit) not previously carried out in such a small baby. ${ }^{1}$ In adolescence staphylococcal endocarditis with an expanding aneurysm associated with the homograft and with the profound neutropenia related to the antibiotics responded to medical and surgical treatment.

We thank Dr Peter Kosovsky for magnetic resonance imaging, Dr Eric F Grabowski and Dr Janice O Gabrilove for use of the granulocyte-stimulating factor, Dr John E O'Loughlin and Dr Michael S Snyder for interpreting the echocardiograms, and Mr Richard Fischer for MRI photography.

1 Ebert PA, Robinson SJ, Stanger P, Engle MA. Pulmonary artery conduits in infants younger than six months of age. $J$ Thorac Cardiovasc Surg 1976;72:351-6.

2 Gabrilove JL, Jakubowski A, Scher H, Sternberg C, Wong G, Grous J, et al. Effect of granulocyte colony-stimulating factor on neutropenia and associated morbidity due to chemotherapy for transitional-cell carcino

3 Bonilla MA, Gillio AP, Rugiero M, Kerman NA, Brochstein Bonilla MA, Gillio AP, Rugiero M, Kerman NA, Brochstein
JA, Abboud M, et al. Effects of recombinant human granulocyte colony stimulating factor on neutropenia in patients with congenital agranulocytosis. $N \mathrm{Engl} \mathrm{J} \mathrm{Med}$ 1989;320:1574-80.

4 Wells WJ. Surgical problems of endocarditis in children. $J$ Cardiac Surg 1989;4:313-6.

5 Alvalos C, Hedzat C, Baldwin GC, Golde DW, Gasson JC DiPersio JF (introduction by Balcerzak SP). Biological activities of human G-CSF and characterization of the human G-CSF receptor [abstract] 29th Meeting of American Society of Hematology, 5-8 December 1987 , Washington DC, page 1 . 